\title{
Kinetics simulation of transmembrane transport of ions and molecules through a semipermeable membrane
}

\author{
S. O. Karakhim ${ }^{1}$ (ID) P. F. Zhuk ${ }^{2}$ - S. O. Kosterin ${ }^{1}$
}

Received: 31 July 2019 / Accepted: 16 December 2019

(C) Springer Science+Business Media, LLC, part of Springer Nature 2020

\begin{abstract}
We have developed a model to study the kinetics of the redistribution of ions and molecules through a semipermeable membrane in complex mixtures of substances penetrating and nonpenetrating through a membrane. It takes into account the degree of dissociation of these substances, their initial concentrations in solutions separated by a membrane, and volumes of these solutions. The model is based on the assumption that only uncharged particles (molecules or ion pairs) diffuse through a membrane (and not ions as in the Donnan model). The developed model makes it possible to calculate the temporal dependencies of concentrations for all processing ions and molecules at system transition from the initial state to equilibrium. Under equilibrium conditions, the ratio of ion concentrations in solutions separated by a membrane obeys the Donnan distribution. The Donnan effect is the result of three factors: equality of equilibrium concentrations of penetrating molecules on each side of a membrane, dissociation of molecules into ions, and Le Chatelier's principle. It is shown that the Donnan distribution (irregularity of ion distribution) and accordingly absolute value of the Donnan membrane potential increases if: (i) the nonpenetrating salt concentration (in one of the solutions) and its dissociation constant increases, (ii) the total penetrating salt concentration and its dissociation constant decreases, and (iii) the volumes ratio increases (between solutions with and without a nonpenetrating substance). It is shown also that only a slight difference between the degrees of dissociation of two substances can be used for their membrane separation.
\end{abstract}

Keywords Membrane transport $\cdot$ Kinetic model $\cdot$ Membrane permeability $\cdot$ Donnan distribution $\cdot$ Donnan potential

\section{Introduction}

The Donnan effect is necessarily taken into account when developing membrane technologies (Sarkar et al. 2010; Steele and Arias 2014; Nouri et al. 2002; Ramirez et al. 2002) because it enables the prediction of ion behaviour in a membrane system and the calculation of the equilibrium concentrations of ions on opposite sides of a semipermeable

S. O. Karakhim

ksa1957@ukr.net; laserlab@biochem.kiev.ua; https://orcid.org/ 0000-0002-8389-0584

P. F. Zhuk

petro_zhuk@ukr.net

S. O. Kosterin

kinet@biochem.kiev.ua

1 Palladin Institute of Biochemistry of the National Academy of Sciences of Ukraine, Kyiv, Ukraine

2 National Aviation University, Kyiv, Ukraine membrane. This effect is also taken into account when explaining the uneven distribution of ions in the cells of living organisms (Lang et al. 2014; Kurbel 2011; Nguyen and Kurtz 2006).

In 1911, Donnan (1995) revealed that if solutions of electrolytes are put into two cells separated by a membrane that is impermeable to at least one type of ions (but permeable to other ions), then, after attainment of thermodynamic equilibrium, the ions are unevenly distributed on each side of the membrane. In this case, the ratio of the concentrations of penetrating $\mathrm{K}^{\mathrm{Z}+}$ cations in solutions from different cells (denoted by indices $i$ and $e$ ) is equal to the inverse ratio of the concentrations of penetrating $\mathrm{A}^{\mathrm{Z}-}$ anions:

$\lambda=\left(\frac{\left[K^{Z+}\right]_{i}}{\left[K^{Z+}\right]_{e}}\right)^{\frac{1}{Z}}=\left(\frac{\left[A^{Z-}\right]_{e}}{\left[A^{Z-}\right]_{i}}\right)^{\frac{1}{Z}}$

This ratio is often called the Donnan equilibrium or Donnan distribution; $\lambda$ is the distribution coefficient.

Taking into account that at equilibrium the electrochemical potentials are the same for each of the common ions in the $i$ - 
and $e$-cells, the following equation can be obtained (Donnan 1924; Davis 2000; Galama et al. 2016; Tian et al. 2015; Mazur et al. 2014):

$E_{D}=\frac{R T}{F} \ln \left(\frac{\left[K^{Z+}\right]_{i}}{\left[K^{Z+}\right]_{e}}\right)^{\frac{1}{Z}}=\frac{R T}{F} \ln \left(\frac{\left[A^{Z-}\right]_{e}}{\left[A^{Z-}\right]_{i}}\right)^{\frac{1}{Z}}=\frac{R T \ln \lambda}{F}$

Here, $R$ is the universal gas constant, $T$ is temperature and $F$ is the Faraday constant.

The Donnan model does not allow for the possibility of the incomplete dissociation of those substances that may be present in the solutions studied. These may be water-soluble proteins capable of counterion binding as well as weak bases (e.g., ammonium hydroxide) and acids of organic and inorganic nature (such as phosphoric, carbonic, nitrous, acetic, citric, tartaric, oxalic, and malic acids) and their salts weakly dissociating into ions.

Within the context of the Donnan model, it is difficult to perform calculations for multicomponent systems in which the volumes of solutions in the $i$ - and $e$-cells are different, thus considerably complicating application of the above model. When developing the Donnan theory, Kosterin and Cherny (1991) took into account that the ratio between volumes of solutions in the $i$ - and $e$-cells may be arbitrary. It turned out that $\lambda$ and $E_{D}$ essentially depend on the ratios of volumes. Under isotonic conditions for membrane vesicles (whose volume is about 0.001 of the incubation medium volume), $E_{D}$ increases by more than twice compared to the "classic" case of equal volumes. One can see from this example how important it is to take into account the ratio between the volumes of solutions separated by a membrane.

To simulate the separation, concentration, removal, and purification of substances, various membrane transport models have been developed. The choice and use of a particular model depends primarily on the process under study and its driving forces, as well as the nature of the semi-permeable membrane used, its structure and the material from which it is made. The consideration of these factors results in a wide variety of models. In the literature, it is possible to find reviews on the application of various models in the description of the most important industrial membrane processes, such as, for example, nanofiltration (Palmeri and Lefebvre 2006; Kumaran and Bajpai 2015), reverse osmosis (Sobana and Panda 2011; Wang et al. 2014; Al-Obaidi et al. 2017), and Donnan dialysis (Pyrzynska 2006) and electrodialysis (Rohman and Aziz 2008). The models based on the NernstPlanck equation (Gimmi and Alt-Epping 2018; FridmanBishop et al. 2018; Galach and Waniewski 2012; Kozmai et al. 2017; Prado-Rubio et al. 2010), extended NernstPlanck equation (Moshtari et al. 2017; Kumaran and Bajpai 2015; Deon et al. 2013), Kedem-Katchalsky equation (Shu et al. 2016; Kim et al. 2010; Tanaka 2012), and also the solution-diffusion model (Yaroshchuk et al. 2011) are considered the most suitable for describing the process of ion separation.

It should be noted that despite the fact that the main purpose of modelling is to calculate the distribution of ions across the membrane, the Donnan approach is not used (sometimes, the Donnan distribution is used only to find the relationship between the concentration of ions in a bulk solution and their concentration in the membrane, membrane pore or nanochannel (Tian et al. 2015; Gimmi and Alt-Epping 2018). This is conditioned by the fact that, as a rule, nonequilibrium processes are studied, and under such conditions it is necessary to consider the membrane potential that is formed during the process of ion separation and its value is unknown since it depends on the distribution of ions, that must be found. As a result, when modelling real processes, researchers are forced to use complex iterative methods.

The simulation of the nanofiltration process (NF) using the extended Nernst-Planck equation (ENP) is described by Palmeri and Lefebvre (2006): "In general, solving the complete system of non-linear algebraic/ordinary differential equations describing ion partitioning and transport in $\mathrm{NF}$ is a relatively involved numerical problem (analytical mathematical solutions exist only for certain single salt solutions). A major difficulty in any numerical treatment comes from the appearance of the unknown ion permeate concentrations in the ENP equations themselves via the filtration condition (2.42), which means that the model must be solved iteratively ... Using an initial guess for $c_{i}^{p}$, the permeate Donnan potential is calculated using Eq. (2.56). This interfacial potential is then be used to obtain the intra-membrane ionic concentrations at the permeate-membrane interface. ... The intra-membrane ionic concentrations at the feed-membrane interface are then used in conjunction with the ion partitioning equations to calculate first the feed Donnan potential, $\Delta \phi^{\mathrm{f}}{ }_{D}$, then the ion feed concentrations, $c_{i}^{f}(\mathrm{cal}$.). If the ion feed concentrations obtained by solving the model, $c_{i}^{f}$ (cal.), agree (to within some preestablished tolerance) with the known values, $c_{i}^{p}$ (real), then the iteration stops and the values used for $c_{i}^{p}$ in the current calculation are the correct numerical solution to the problem. If, on the other hand, $c_{i}^{f}(\mathrm{cal}$. $) \neq c_{i}^{f}$ (real), then new starting values are chosen for $c_{i}^{p}$ and the iteration continues".

As mentioned above, since the Donnan model is a thermodynamic one, it is applicable only under equilibrium conditions and does not enable the process kinetics to be calculated. However, when developing the membrane technologies for concentration, separation, isolation or removal of different substances, the need to study kinetic dependencies is clear (Tanaka 2012; Pyrzynska 2006; Kozmai et al. 2017; Grzegorczyn and Ślęzak 2006), since temporal dependencies of concentrations are primary experimental data.

To undertake such research, some other approaches beyond the Donnan model have been developed. These are based on 
application of the Nernst-Planck equation (Cohen and Cooley 1965; Zhao et al. 2012; Higa and Kira 1992; Szczepański and Szczepańska 2017) which is one of expressions of linear phenomenological laws in nonequilibrium thermodynamics (Pyrzynska 2006). However, in our opinion, it does not seem appropriate to use the equations of nonequilibrium thermodynamics in which the flows are linear functions of forces to describe the kinetic dependencies. Such equations can be applicable only in the area close to equilibrium, where the linear dependence between the forces and flows can hold (Kondepudi and Prigogine 1998, page 337). There have therefore not been any adequate models and theories developed to investigate kinetic dependencies.

Our approach is kinetic, rather than thermodynamic. Therefore, it is free of limitations that are peculiar to thermodynamic models and allows the direct calculation of kinetic dependences for each ion and each molecule from the very beginning of the separation of substances through a semipermeable membrane to equilibrium. This is important because, as mentioned earlier, the beginning of a process in which the system is far from equilibrium is attracting the interest of researchers (Kozmai et al. 2017).

Our approach is aimed at simplifying the calculation of the transmembrane transport of ions and molecules due to the concentration gradient so that it would be possible to quickly and easily calculate their concentrations in multicomponent solutions separated by a membrane, at any time until the system reaches an equilibrium state, based only on the initial concentrations of soluble substances. Knowing the ion distribution, one can also calculate the Donnan potential.

This simplification is achieved by improving our understanding of the mechanism of the penetration of substances through a semipermeable membrane. Thanks to this, it was possible to create an algorithm that does not require either the membrane potential (as in the Donnan approach) or the equilibrium distribution of substances (which is required under the thermodynamic approach) to be considered, but rather allows kinetic calculations to be employed knowing only the initial concentrations of the substances involved in the investigated process. As a result, significant difficulties can be avoided in numerical calculations even with a substantial increase in the amount of substances involved in the membrane process under study.

The proposed kinetic approach can be considered as an extension of the scope of the Donnan approach from equilibrium to non-equilibrium conditions. It is necessary to consider two states, i.e. the initial and equilibrium state, in order to describe the membrane system under the thermodynamic Donnan approach, while the kinetic approach requires only the initial state to be considered. To start the process of particle distribution through the membrane, it is also necessary to set nonzero permeability coefficients for penetrating molecules. The result of the calculation is not only the equilibrium distribution of ions and molecules in solutions on both sides of the semipermeable membrane, but also their distribution at any moment in time.

In this work, we present a kinetic model that we have developed that enables calculation of the (i) temporal variation of concentrations of all ions and molecules present in a system and (ii) the Donnan equilibrium $\lambda$ (and, correspondingly, the Donnan membrane potential $E_{D}$ ) for composite mixtures of substances penetrating and nonpenetrating through a membrane. The kinetic model takes into account the degree of dissociation of these substances, their initial concentrations in solutions separated by a membrane, and the volumes of these solutions.

\section{A model taking into account the penetration of molecules through a membrane}

The Donnan model becomes problematic when calculating multicomponent mixtures involving the incomplete dissociation of the substances involved and unequal volumes of solutions separated by a membrane. In addition, there is no possibility to analyse the kinetic dependencies. Therefore, to address this, we have developed a model based on another principle. Contrary to the Donnan model (in which it is supposed that separate ions penetrate through a membrane), our model is based on the supposition that uncharged particles (molecules or ion pairs) penetrate through a membrane (Pyrzynska 2006; Duffey et al. 1978; Marcus and Hefter 2006; Johnson and Pytkowicz 1978; Osterhout 1925). The penetration mechanism is simple diffusion. The diffusion of molecules or ion pairs through a membrane does not violate the electroneutrality of solutions and is described by the Fick equation.

The observation of electroneutrality in the Donnan model may in fact be interpreted as the membrane permeability for molecules (Philipse and Vrij 2011), since cations and anions formed during the dissociation of molecules are transported through the membrane in equivalent amounts according to the stoichiometry of their parent molecules. It should be also noted that the effect of an electric field near a membrane on the permeability of neutral molecules must be much less than on the permeability of ions. Therefore, in our kinetic model we did not take into account the effect of membrane potential on the penetration of molecules through a membrane.

Contrary to the classic Donnan model, in our model we take into account the dissociation of molecules into ions. The solutions are therefore considered as a mixture of ions (having an electric charge) and undissociated molecules or ion pairs (being electrically neutral). It is hereupon considered that not only are individual ions nonpenetrating through a membrane but also all molecules or ion pairs (neutral compounds) containing these ions (salts, acids or bases that could be formed in the system studied) as well. 
As for the Donnan model, we shall present, for the sake of simplicity, our kinetic model in terms of concentrations rather than activities. We suppose that all solutions are ideal; however, it is taken into account that the volumes of solutions separated by a membrane may differ.

Let us consider a system similar to that analysed by Kosterin and Cherny (1991) in which two solutions put into the $i$ - and $e$-cells and separated by a semipermeable membrane of the area $S$ have volumes $V_{i}$ and $V_{e}$, respectively. Initially, the solution in the $e$-cell contains substance $\mathrm{KB}$ of concentration $C_{1}$ and salt KA of concentration $C_{2}$, while the solution in the $i$ cell contains only salt KA of concentration $C_{3}$. Substance KB cannot penetrate through a membrane because of the large size of the $\mathrm{B}^{-}$anion, while substance KA can diffuse through this membrane. Both compounds can dissociate into ions:

$$
\begin{aligned}
& k_{1} \\
& K B \underset{k_{-1}}{\longrightarrow} K^{+}+B \\
& k_{2} \\
& K A \underset{k_{-2}}{\underset{\longrightarrow}{\longrightarrow} K^{+}}+A^{-}
\end{aligned}
$$

Here, $k_{1}$ and $k_{-1}$ are the rate constants, respectively, of the direct $\left(\mathrm{s}^{-1}\right)$ and reverse $\left(\mathrm{M}^{-1} \mathrm{~s}^{-1}\right)$ reactions of dissociation of nonpenetrating substance $\mathrm{KB}$ into ions; $k_{2}$ and $k_{-2}$ are the rate constants, respectively, of the direct $\left(\mathrm{s}^{-1}\right)$ and reverse $\left(\mathrm{M}^{-1} \mathrm{~s}^{-1}\right)$ reactions of the dissociation of penetrating salt $\mathrm{KA}$ into ions.

The corresponding dissociation constants $\left(K_{1}\right.$ and $\left.K_{2}\right)$ may be written as:

$K_{1}=\frac{\left[K^{+}\right]\left[B^{-}\right]}{[K B]}=\frac{k_{1}}{k_{-1}}$

$K_{2}=\frac{\left[K^{+}\right]\left[A^{-}\right]}{[K A]}=\frac{k_{2}}{k_{-2}}$

If salt KA is a strong electrolyte, then Eqs. (4) and (6) may be considered as the dissociation of the $\mathrm{K}^{+} \mathrm{A}^{-}$ion pair.

It should be noted that concentrations $[K B]$ and $[K A]$ represent the undissociated amount of molecules of the corresponding substances (or the amount of ions united into ion pairs) rather than their initial concentration. The initial concentrations of substances may be presented as:

$C_{1}=[K B]_{e}+\left[B^{-}\right]_{e}$

$C_{2}=[K A]_{e}+\left[A^{-}\right]_{e}$

$C_{3}=[K A]_{i}+\left[A^{-}\right]_{i}=[K A]_{i}+\left[K^{+}\right]_{i}$

As a result of the penetration of KA molecules (i.e., their undissociated amount $[K A]$ ) through a membrane, the considered system begins to move from the initial state to equilibrium. The latter will occur when $[K A]$ concentrations in both solutions become equal (i.e., when the chemical potentials for undissociated KA molecules in solutions separated by a membrane become equal):

$[K A]_{e}=[K A]_{i}$

The mathematical model of this process is a system of differential equations:

$\frac{d[K A]_{e}}{d t}=\left\{-D_{K A}\left([K A]_{e}-[K A]_{i}\right) \frac{S}{V_{e}}\right\}-k_{2}[K A]_{e}$

$$
+k_{-2}\left[K^{+}\right]_{e}\left[A^{-}\right]_{e}
$$

$\frac{d[K A]_{i}}{d t}=\left\{D_{K A}\left([K A]_{e}-[K A]_{i}\right) \frac{S}{V_{i}}\right\}-k_{2}[K A]_{i}$

$+k_{-2}\left[K^{+}\right]_{i}\left[A^{-}\right]_{i}$

$\frac{d[K B]_{e}}{d t}=\left\{-D_{K B}\left([K B]_{e}-[K B]_{i}\right) \frac{S}{V_{e}}\right\}-k_{1}[K B]_{e}$

$$
+k_{-1}\left[K^{+}\right]_{e}\left[B^{-}\right]_{e}
$$

$\frac{d[K B]_{i}}{d t}=\left\{D_{K B}\left([K B]_{e}-[K B]_{i}\right) \frac{S}{V_{i}}\right\}-k_{1}[K B]_{i}$

$$
+k_{-1}\left[K^{+}\right]_{i}\left[B^{-}\right]_{i}
$$

$\frac{d\left[A^{-}\right]_{e}}{d t}=k_{2}[K A]_{e}-k_{-2}\left[K^{+}\right]_{e}\left[A^{-}\right]_{e}$

$\frac{d\left[A^{-}\right]_{i}}{d t}=k_{2}[K A]_{i}-k_{-2}\left[K^{+}\right]_{i}\left[A^{-}\right]_{i}$

$\frac{d\left[K^{+}\right]_{e}}{d t}=k_{2}[K A]_{e}-k_{-2}\left[K^{+}\right]_{e}\left[A^{-}\right]_{e}$

$$
+k_{1}[K B]_{e}-k_{-1}\left[K^{+}\right]_{e}\left[B^{-}\right]_{e}
$$

$\frac{d\left[K^{+}\right]_{i}}{d t}=k_{2}[K A]_{i}-k_{-2}\left[K^{+}\right]_{i}\left[A^{-}\right]_{i}$

$$
+k_{1}[K B]_{i}-k_{-1}\left[K^{+}\right]_{i}\left[B^{-}\right]_{i}
$$

$\frac{d\left[B^{-}\right]_{e}}{d t}=k_{1}[K B]_{e}-k_{-1}\left[K^{+}\right]_{e}\left[B^{-}\right]_{e}$

$\frac{d\left[B^{-}\right]_{i}}{d t}=k_{1}[K B]_{i}-k_{-1}\left[K^{+}\right]_{i}\left[B^{-}\right]_{i}$

Here, $D_{K A}$ is the permeability coefficient of the membrane to KA molecules ( $\mathrm{dm} \cdot \mathrm{s}^{-1}$ ), while $D_{K B}$ is the permeability coefficient of the membrane to KB molecules $\left(\mathrm{dm} \cdot \mathrm{s}^{-1}\right)$.

Differential equations must be written for each molecular or ion particle present in the $e$ - and $i$-solutions. In this case, one should take into account that the ions formed due to the dissociation of substances present in the system may unite in any combination forming molecules of substances that were not present in the system initially. Variation of the concentrations of these substances formed in the solution also has to be taken into account when setting up a system of differential equations. 
The numerical solution of this system of equations enables the temporal variation of the concentration of each reaction component to be calculated. As mentioned above, the process proceeds until the concentrations of the undissociated part of molecules of penetrating salt on each side of the membrane become equal. In this case, all flows stop and the system reaches the state of equilibrium. An analysis of the presented system of differential equations shows that its solution is unique and stable.

The system of Eqs. (11-20) is a special case of differential equations on graphs considered by Volpert and Hudyaev (1975) in the analysis of chemical kinetics equations (Chapter 12, "Chemical Kinetics Equations"). Therefore, our analysis of the system of differential Eqs. (11-20) is based on the results obtained by Volpert and Hudyaev. Theorem ( $\$ 3$, item 2 "Acyclic Graph") suggests the existence of a unique non-negative solution to the system of differential Eqs. (1120 ) on the entire time semi-axis (from 0 to $\infty$ ), which has a limit as $t \rightarrow \infty$. This limit characterizes the equilibrium state of the system and is stable with respect to changes in the initial conditions, as it appears from the theorem ( $\S 3$, item 4 , "Reversible Reactions"). These properties of the solution are retained even with an increase in the number of system components, since in this case the conditions of the above theorems are also fulfilled (Volpert and Hudyaev 1975).

At equilibrium, the ratios between the concentrations of $\mathrm{K}^{+}$ and $\mathrm{A}^{-}$ions in solutions separated by a membrane obey the Donnan ratio Eq. (1) (as may be seen from Table 1). For comparison, the results obtained by Kosterin and Cherny (1991) within the context of the classic Donnan model under the identical initial conditions are presented in Table 1.
In the classic Donnan model, first the Donnan ratio (Eq. (1)) is obtained thermodynamically, and then the equilibrium concentrations of ions are calculated using Eq. (1).

Within the context of our kinetic model, we not speculate as to the ratios between the penetrating ions after the system reaches equilibrium. The proposed model enables the calculation of the concentrations of all substances (ions and molecules) in the process of system transition from the initial state to equilibrium. Only at the end of the calculations (when the concentrations of all ions and molecules stop changing and the system reaches equilibrium), on the basis of the equilibrium concentrations of penetrating ions, does it become possible to calculate their ratios from Eq. (1) and to check if they are distributed in accordance with those predicted by the classic Donnan model. With the calculated equilibrium concentrations of penetrating ions, it is possible also to calculate the Donnan potential using Eq. (2).

Our kinetic model thereby enables the equilibrium concentrations of molecules and ions to be directly calculated. The results of such a calculation confirm that at equilibrium the penetrating ions are distributed as predicted by the classic Donnan theory (Table 1).

It is possible to prove, using the Donnan thermodynamic approach, the existence of the same equilibrium state both for the classical Donnan model and model we have developed (see Appendix). On the one hand, this may suggest that the assumptions that form the basis of the Donnan model and our model can be fulfilled simultaneously. On the other hand, this fact can be regarded as proof of the equivalence of our kinetic model to the Donnan model, which allows us to use the approach proposed in this article for modelling membrane processes.
Table 1 Equilibrium concentrations of ions and molecules (in $\mathrm{mM}$ ) and the Donnan distribution $\lambda$ at different volume ratios $\theta=V_{i} / V_{e}$ : a comparison of the results obtained when solving the system of differential Eqs. (11-20) (at a high degree of dissociation of substances $\mathrm{KB}$ and $\mathrm{KA}-K_{1}=K_{2}=1000 \mathrm{M}$ and impermeability to membrane for substance $\mathrm{KB}-D_{K B}=0 ; S=1 \mathrm{dm}^{2}$ ) with those obtained by Kosterin and
Cherny (1991) within the framework of the classic Donnan model from Eqs. (13) and (15-17) in (Kosterin and Cherny 1991) under the same initial conditions $\left(C_{1}=285 \mathrm{mM} ; C_{2}=15 \mathrm{mM} ; C_{3}=300 \mathrm{mM}\right)$. The Donnan potential was calculated at $T=300 \mathrm{~K}(R=8.314 \mathrm{~J} / \mathrm{mol} \cdot \mathrm{K}$, $F=96,490 \mathrm{C} / \mathrm{mol}$ )

\begin{tabular}{|c|c|c|c|c|}
\hline \multirow[t]{2}{*}{$\begin{array}{l}\text { Equilibrium concentrations } \\
\text { of ions and molecules }\end{array}$} & \multicolumn{2}{|c|}{$\begin{array}{l}\text { Results of solving the system of } \\
\text { Eqs. (11-20) }\end{array}$} & \multicolumn{2}{|c|}{$\begin{array}{l}\text { Results of calculation from Eqs. (13, 15-17) } \\
\text { in (Kosterin and Cherny 1991) }\end{array}$} \\
\hline & $\theta=1$ & $\theta=0.001$ & $\theta=1$ & $\theta=0.001$ \\
\hline$\left[K^{+}\right]_{e}$ & 393.3 & 300.1 & 393.4 & 300.2 \\
\hline$\left[A^{-}\right]_{e}$ & 108.4 & 15.2 & 108.4 & 15.2 \\
\hline$\left[K^{+}\right]_{i}=\left[A^{-}\right]_{i}$ & 206.5 & 67.6 & 206.6 & 67.6 \\
\hline$[K A]_{i}=[K A]_{e}$ & 0.043 & 0.0046 & 0 & 0 \\
\hline$[K B]_{e}$ & 0.1 & 0.086 & 0 & 0 \\
\hline$\left[B^{-}\right]_{e}$ & 284.9 & 284.9 & 285.0 & 285.0 \\
\hline$\lambda=\left[A^{-}\right]_{e} /\left[A^{-}\right]_{i}$ & 0.525 & 0.225 & 0.525 & 0.225 \\
\hline$\lambda=\left[K^{+}\right]_{i} /\left[K^{+}\right]_{e}$ & 0.525 & 0.225 & 0.525 & 0.225 \\
\hline$E_{D}, \mathrm{mV}$ & -16.7 & -38.6 & -16.7 & -38.6 \\
\hline
\end{tabular}


The system of differential Eqs. (11-20) can be solved numerically with, e.g., mathematical program packages such as "MatLab" or "Maple" or using the independently developed (algorithmic) programs with programming languages. However, it is much simpler and more convenient to use COPASI problem-oriented software (a complex pathway simulator, copasi.org), which provides for the possibility of the existence of several compartments. Furthermore, it is enough to write down processes of the dissociation of molecules and their diffusion through the membrane in the form of chemical equations instead of a system of differential equations. COPASI automatically converts the reaction network to a set of differential equations.

The experimental temporal dependencies obtained for a variety of concentrations of substances participating in the process under study may be analysed by fitting the experimental data to a model defined by differential equations for the determination of the kinetic parameters of model.

Three important points should be noted.

The system of Eqs. (11-20) is written for the case when both substances $\mathrm{KB}$ and KA present in the system penetrate through a membrane, with their penetrating speed determined by the corresponding permeability coefficients. If the permeability coefficient of the given substance is zero, then this means that this substance cannot penetrate through the membrane.

When beginning calculations, the initial concentrations must be presented with an allowance made for dissociation. This means that firstly it is necessary to calculate the concentrations of ions and of undissociated molecules for solutions in each cell. This is of particular importance when kinetic parameters of the model are determined. If the equilibrium concentrations are calculated, then the total concentrations for molecules (in this case, $C_{1}, C_{2}$ and $C_{3}$ ) and zero concentrations for ions may be used as the initial concentrations.

If there are no common ions in the salts solutions separated by the membrane, one should take into account that hydrogen and hydroxyl ions are present in aqueous solutions. These are capable of producing molecules of acids and alkalis with the ions that result from the dissociation of salts. Along with salt molecules, acid and alkali molecules also can penetrate through the membrane.

One should therefore take into account all the above factors when setting up the system of differential equations.

\section{Nature of the Donnan distribution}

Taking into account the process of the dissociation of molecules makes it possible to understand more clearly the nature of the Donnan distribution, i.e., to understand why the equilibrium distribution of penetrating ions is described by Eq. (1). This is determined by three factors related to undissociated molecules. These factors are as follows: (i) equality of the equilibrium concentrations of penetrating molecules on each side of the membrane, (ii) molecules dissociating into ions, and (iii) Le Chatelier's principle. As a result of the latter, the uniform distribution of ions of the penetrating substance is disrupted due to the common ions of the nonpenetrating substance present in the solution.

Let us consider each of the three above factors in more detail.

\section{Equality of equilibrium concentrations of penetrating undissociated molecules on each side of a membrane}

As was shown above (see Table 1), if a membrane is permeable to molecules (rather than ions), then the state of equilibrium occurs when the concentrations of undissociated molecules (penetrating through a membrane) in the $i$ - and $e$-solutions become equal. As a result, their flows through a membrane in the opposite directions become equal.

The classic Donnan model takes into account only ions; the concentrations of molecules (because of their complete dissociation into ions) are zero, in particular, at equilibrium. However, the fact that concentrations of molecules are zero may be considered as the limiting case of dissociation when the dissociation constants are infinite.

No matter what the particles penetrating through a membrane are - undissociated molecules, ions or simultaneously ions and molecules (with electroneutrality) - at equilibrium the equality of concentrations of penetrating undissociated molecules holds:

$\frac{[K A]_{i}}{[K A]_{e}}=\frac{\left[K^{+}\right]_{i}\left[A^{-}\right]_{i}}{\left[K^{+}\right]_{e}\left[A^{-}\right]_{e}}=1$

If in solutions there are several kinds of molecules penetrating through a semipermeable membrane, then at equilibrium the equality of concentrations of penetrating undissociated molecules on each side will hold for molecules of all kinds.

\section{Dissociation of molecules into ions}

It is evident that electrolytic dissociation is necessary for the Donnan effect to occur. Due to the dissociation of molecules in accordance with Eqs. (5) and (6), it is not only ions that appear in solution. The products of cation and anion concentrations also appear in the equations. The equality of these products in different cells results from equal equilibrium distributions of undissociated molecules in the given cells as well as from equal dissociation constants of penetrating substances of the given type in solutions separated by a semipermeable membrane.

To clearly see how the Donnan distribution, dissociation and equality of equilibrium concentrations of undissociated 
molecules in solutions separated by a semipermeable membrane are interrelated, let us present Eq. (21) as

$$
\left[K^{+}\right]_{i}\left[A^{-}\right]_{i}=\left[K^{+}\right]_{e}\left[A^{-}\right]_{e}
$$

and Eq. (6) separately for solutions in the $i$ - and $e$-cells:

$$
\begin{aligned}
K_{2} & =\frac{\left[K^{+}\right]_{i}\left[A^{-}\right]_{i}}{[K A]_{i}} \\
K_{2} & =\frac{\left[K^{+}\right]_{e}\left[A^{-}\right]_{e}}{[K A]_{e}}
\end{aligned}
$$

It follows from these equations that if ions are distributed in accordance with the Donnan effect (i.e., Eq. (22) holds), then the equality $[K A]_{e}=[K A]_{i}$ also has to be true, since the numerators in Eqs. (23) and (24) are equal (Osterhout 1929). Moreover, vice versa, if the equilibrium concentrations of undissociated penetrating salts are the same, then the Donnan Eq. (22) must hold.

\section{Le Chatelier's principle}

However, one should note that a third factor is required to reveal the Donnan effect (non-uniform distribution of ions), namely Le Chatelier's principle, which is related to the presence of common ions of the substances penetrating and nonpenetrating through a membrane. The electrolytic dissociation of molecules into ions per se can provide only uniform distribution of ions $\left[K^{Z+}\right]_{i}=\left[K^{Z+}\right]_{e}$ and $\left[A^{Z-}\right]_{i}=\left[A^{Z-}\right]_{e}$ (i.e., $\lambda=1$ ) in solutions separated by a membrane (see Table 2 , columns 2 and 5). The presence of an additional number of $\mathrm{K}^{+}$ions in the $i$-cell due to dissociation of substance $\mathrm{KB}$

Table 2 Equilibrium distribution of ions and undissociated molecules (in $\mathrm{mM}$ ) in solutions separated by a semipermeable membrane $\left(V_{i}=V_{e}\right)$ in

\begin{tabular}{|c|c|c|c|c|c|}
\hline $\begin{array}{l}\text { Equilibrium concentrations } \\
\text { of ions and molecules } \\
\text { (in } \mathrm{mM} \text { ) }\end{array}$ & $\begin{array}{l}C_{3}=0.3 \mathrm{M} \\
C_{1}=0 \mathrm{M} \\
K_{1}=0 \\
K_{2}=10 \mathrm{M}\end{array}$ & $\begin{array}{l}C_{3}=0.3 \mathrm{M} ; \\
C_{1}=0.3 \mathrm{M} ; K_{1}=0.1 \\
\mathrm{M} ; \\
K_{2}=10 \mathrm{M}\end{array}$ & $\begin{array}{l}C_{3}=0.3 \mathrm{M} ; \\
C_{1}=0.3 \mathrm{M} ; K_{1}=10 \\
\mathrm{M} ; \\
K_{2}=10 \mathrm{M}\end{array}$ & $\begin{array}{l}C_{3}=0.3 \mathrm{M} \\
C_{1}=0 \mathrm{M} \\
K_{1}=0 \\
K_{2}=0.1 \mathrm{M}\end{array}$ & $\begin{array}{l}C_{3}=0.3 \mathrm{M} ; \\
C_{1}=0.3 \mathrm{M} ; K_{1}=0.1 \\
\mathrm{M} ; \\
K_{2}=0.1 \mathrm{M}\end{array}$ \\
\hline 1 & 2 & 3 & 4 & 5 & 6 \\
\hline$\left[K^{+}\right]_{e}$ & 147.8 & 220.6 & 386.7 & 82.3 & 163.3 \\
\hline$\left[A^{-}\right]_{e}$ & 147.8 & 127.0 & 97.9 & 82.3 & 49.3 \\
\hline$\left[K^{+}\right]_{i}=\left[A^{-}\right]_{i}$ & 147.8 & 167.4 & 194.5 & 82.3 & 89.7 \\
\hline$[K A]_{i}=[K A]_{e}$ & 2.2 & 2.8 & 3.8 & 67.7 & 80.5 \\
\hline$C_{i}=[K A]_{i}+\left[A^{-}\right]_{i}$ & 150.0 & 170.2 & 198.3 & 150.0 & 170.2 \\
\hline$C_{e}=[K A]_{e}+\left[A^{-}\right]_{e}$ & 150.0 & 129.8 & 101.7 & 150.0 & 129.8 \\
\hline$[K B]_{e}$ & 0 & 206.4 & 11.2 & 0 & 186.0 \\
\hline$\left[B^{-}\right]_{e}$ & 0 & 93.6 & 288.8 & 0 & 114.0 \\
\hline$\lambda=\left[A^{-}\right]_{e}\left[A^{-}\right]_{i}$ & 1.000 & 0.759 & 0.503 & 1.000 & 0.55 \\
\hline$\left.\lambda=\left[K^{+}\right]_{i} \bigwedge K^{+}\right]_{e}$ & 1.000 & 0.759 & 0.503 & 1.000 & 0.55 \\
\hline
\end{tabular}
the presence and absence of nonpenetrating substance KB (with concentration $C_{1}$ ) in the solution in the $e$-cell at different dissociation cannot change the equilibrium constant $K_{2}$ characterizing the dissociation of salt KA. However, this causes the redistribution of ions in the $i$-cell, thus leading to a disturbance of the uniform distribution of ions in solutions separated by a membrane (i.e., to the Donnan effect when $\lambda>1$ ).

As the total concentration of $\mathrm{K}^{+}$ions increases, the equilibrium shifts (in accordance with Le Chatelier's principle) towards increasing the concentration of undissociated KA molecules due to binding the $\mathrm{A}^{-}$counterion. As a result, the concentration of $\mathrm{A}^{-}$decreases just as much as the concentration of $\mathrm{K}^{+}$increases (relative to the concentrations of ions in the adjacent cell).

A shift in equilibrium due to Le Chatelier's principle is clearly seen from the results presented in Table 2 . This shows the results of the equilibrium distribution of ions and undissociated molecules in solutions separated by a semipermeable membrane in the presence and absence of a nonpenetrating substance. If a nonpenetrating substance $\mathrm{KB}$ is present in the system, then the equilibrium concentration of undissociated molecules of penetrating substance KA is always higher than in the case of a lack of substance KB (e.g., in column 4, the equilibrium concentration is almost twice as big as in column 1). This indicates that adding substance $\mathrm{KB}$ (which is a source of $\mathrm{K}^{+}$cations) shifts the equilibrium towards an increase in the concentration of undissociated KA molecules. Moreover, this gives rise to their concentration in both solutions separated by a membrane, as compared with a situation when there was no nonpenetrating substance in the system.

On the other hand, due to transferring substance KA into solution in the $e$-cell (with subsequent dissociation), reaction equilibrium (Eq. (3) also shifts in accordance with Le Chatelier's principle. One can calculate the concentrations of

constants of substance $\mathrm{KB}\left(K_{1}\right)$ and penetrating through membrane substance KA $\left(K_{2}\right)$. The initial (total) concentration of substance KA in the solution in the $i$-cell $-C_{3}=0.3 \mathrm{M}\left(C_{2}=0\right)$ 
ions and undissociated molecules in solution from the $e$-cell in the initial state (at $C_{1}=0.3 \mathrm{M}$ and $K_{1}=0.1 \mathrm{M}$ ). The concentration of $\mathrm{K}^{+}$and $\mathrm{B}^{-}$ions is $130.3 \mathrm{mM}$, while that of the undissociated amount of $\mathrm{KB}$ molecules is $169.7 \mathrm{mM}$. One can see from Table 2 (columns 3 and 6) that if substance KA is present in solution in the $e$-cell, then the concentration of the undissociated amount of KB molecules increases considerably.

The presence of common ions of the substances penetrating and nonpenetrating through the membrane therefore leads to an increase in the concentrations of undissociated molecules of those substances due to Le Chatelier's principle. In this case, the concentration of common ions increases, while that of counterions decreases. This results in an irregular distribution of ions in solutions separated by a membrane, i.e., in the Donnan effect.

One can see from Table 2 that if nonpenetrating substance $\mathrm{KB}$ is present in the system, then, at equilibrium, substance $\mathrm{KA}$ is irregularly distributed in the solutions on either side of the membrane $\left(C_{i}>C_{e}\right)$.

When comparing the results presented in columns 3 and 6 of Table 2, one should note that the equilibrium distributions $\lambda$ of ions differ considerably, although the concentrations of substances $\mathrm{KA}$ and $\mathrm{KB}$ in the initial states were equal $\left(C_{1}=C_{3}=0.3 \mathrm{M}\right)$. Moreover, the amount of substance KA that has moved from the $i$-cell into the $e$-cell is the same too (compare the $C_{i}$ and $C_{e}$ values). In the case under study, the difference in the equilibrium distribution of ions is due to the dissociation constants of the penetrating substance.

When analysing the equilibrium distribution of substances, ions and undissociated molecules in solutions separated by a membrane, one can see that only the undissociated amount of molecules of substances penetrating through a membrane at equilibrium is distributed regularly, both in the presence and absence of nonpenetrating substances in the system. One may see in this the demonstration of the more fundamental nature of undissociated molecules as compared to ions in the membrane processes.

The Donnan effect is thereby the result of three factors, namely, (i) the equality of equilibrium concentrations of penetrating molecules on each side of the membrane, (ii) the electrolytic dissociation of molecules, and (iii) Le Chatelier's principle related to the presence of common ions in substances penetrating and nonpenetrating through a membrane.

\section{Effect of dissociation constants of salts on the Donnan distribution}

One can see from Table 1 that at equilibrium both our kinetic model and the classic Donnan model lead to the same results. However, this is true only in the case of relatively high dissociation constants. Their variation essentially influences both the Donnan distribution and the $E_{D}$ value (Table 3 ).

A reduction in the dissociation constant of nonpenetrating molecules leads to a decrease both in the Donnan distribution ( $\lambda$ approaches one) and the absolute value of $E_{D}$ due to a decrease in the concentration of nonpenetrating ions responsible for the irregular distribution of penetrating ions.

Furthermore, a decrease in the dissociation constant of penetrating salt (ion pair) leads to a considerable increase in the Donnan distribution (growth of ion distribution irregularity) and an increase of the absolute value of $E_{D}$ since a reduction of $K_{2}$ is equivalent to a decrease of the KA concentration in the Donnan model. These effects are clearly seen when comparing the data from Table 1 and Table 3.

Hence the Donnan distribution $\lambda$ (irregularity of ion distribution) and accordingly absolute value of the Donnan membrane potential $E_{D}$ increases if: (i) the nonpenetrating salt concentration
Table 3 Effect of dissociation constants of penetrating $\left(K_{2}\right)$ and nonpenetrating $\left(K_{1}\right)$ substances at different volume ratios $\theta=V_{i} / V_{e}$ on the values of the Donnan distribution $\lambda$ and membrane potential $E_{D}$ under isotonic conditions at different initial concentrations (all concentrations are given in $\mathrm{mM}$ ). The Donnan potential was calculated at $T=300 \mathrm{~K}$ $(R=8.314 \mathrm{~J} / \mathrm{mol} \cdot \mathrm{K}, F=96,490 \mathrm{C} / \mathrm{mol}) . S=1 \mathrm{dm}^{2}$

\begin{tabular}{|c|c|c|c|c|c|}
\hline \multirow[t]{2}{*}{ Equilibrium concentrations of ions and molecules } & \multicolumn{2}{|c|}{$\begin{array}{l}C_{1}=285 \mathrm{mM} ; K_{1}=1 \mathrm{mM} \\
C_{2}=15 \mathrm{mM} ; K_{2}=10^{3} \mathrm{M} \\
C_{3}=300 \mathrm{mM}\end{array}$} & \multicolumn{2}{|c|}{$\begin{array}{l}C_{1}=285 \mathrm{mM} ; K_{1}=10^{3} \mathrm{M} \\
C_{2}=15 \mathrm{mM} ; K_{2}=1 \mathrm{mM} \\
C_{3}=300 \mathrm{mM}\end{array}$} & \multirow{2}{*}{$\begin{array}{l}C_{1}=495 \mathrm{mM} ; K_{1}=10^{3} \mathrm{M} \\
C_{2}=5 \mathrm{mM} ; K_{2}=17.5 \mu \mathrm{M} \\
C_{3}=500 \mathrm{mM} \\
\theta=0.001\end{array}$} \\
\hline & $\theta=1$ & $\theta=0.001$ & $\theta=1$ & $\theta=0.001$ & \\
\hline$\left[K^{+}\right]_{e}$ & 158.8 & 25.9 & 285.4 & 285.0 & 494.8 \\
\hline$\left[A^{-}\right]_{e}$ & 157.0 & 15.3 & 0.5 & 0.05 & 0.0002 \\
\hline$\left[K^{+}\right]_{i}=\left[A^{-}\right]_{i}$ & 157.9 & 19.9 & 12.3 & 3.9 & 0.3 \\
\hline$[K A]_{i}=[K A]_{e}$ & 0.025 & 0.0004 & 151.1 & 15.2 & 5.5 \\
\hline$[K B]_{e}$ & 283.2 & 274.4 & 0.08 & 0.08 & 0.2 \\
\hline$\left[B^{-}\right]_{e}$ & 1.8 & 10.6 & 284.9 & 284.9 & 494.8 \\
\hline$\left.\lambda=\left[A^{-}\right]_{e} \int A^{-}\right]_{i}$ & 0.994 & 0.768 & 0.043 & 0.014 & 0.0006 \\
\hline$\left.\lambda=\left[K^{+}\right]_{i} \backslash K^{+}\right]_{e}$ & 0.994 & 0.768 & 0.043 & 0.014 & 0.0006 \\
\hline$E_{D}, \mathrm{mV}$ & -0.16 & -6.8 & -81 & -110 & -192 \\
\hline
\end{tabular}


in one of the solutions and its dissociation constant increases, (ii) the total penetrating salt concentration and its dissociation constant decreases, and (iii) the ratio decreases between the volumes of solution in which nonpenetrating salt is absent and solution containing the nonpenetrating substance.

If a penetrating substance with a dissociation constant $K_{2}=0.0175 \mathrm{mM}$ (characteristic of the dissociation of acetic acid, i.e., in this case HAc is a penetrating substance KA) and higher concentrations are used, then it is possible to reach under isotonic conditions a sufficiently high membrane potential of $190 \mathrm{mV}$ (Table 3, the last column).

A possibility to reach such high potentials (compared to the maximum potentials observed in living cells) can be applied when calibrating potential-sensitive probes. Vesicles are the usual model system for performing such calibrations. The potentials of a desired value can be obtained on their membrane (Mazur 2014). These potentials are achieved under conditions when the equilibrium concentrations of ions do not change for a long time. This makes it possible to measure the membrane potential and corresponding fluorescence of a potentialdependent probe with sufficient accuracy. Our model enables the calculation of the conditions for obtaining potentials of a desired value at the membranes of vesicles.

If equilibrium is reached rather slowly, then our model can be also applied to determine the point of time when the studied membrane potential $E_{D}$ or ion concentration differ from their equilibrium values by a certain value, e.g., comparable with the experimental accuracy (by $1 \%$ or $5 \%$ ). By way of example, one may consider the investigations by Vega et al. (2010) dealing with the determination of concentrations of free ions in natural systems where the authors use $t_{95 \%}$, i.e., the time required for free ions to reach $95 \%$ of the equilibrium concentrations.

Nelhof and Sollner (1957) showed that when along with nonpenetrating ions, several penetrating ions (whose penetrating speeds through a membrane differ considerably) are present in the membrane system, then, at some point of time, the concentration of penetrating ions of one type may be much bigger or smaller than its concentration at equilibrium. The presence of such effects has been established experimentally by the authors. Our kinetic model may be very useful in such cases, not only for determining the point of time at which the maximum or minimum concentration of given ions is reached, but also for "designing" and analyzing similar systems in which it is possible (i) to reach higher separation than at equilibrium, and (ii) to save time since optimal conditions for the separation of substances are achieved much faster than equilibrium (see, for example, Fig. 1).

The proposed kinetic model can be used to calculate the permeability coefficients of substances through the membrane in diffusion dialysis. Similar studies were performed by Luo et al. (2013) to simulate the recovery of inorganic acids from metal treatment wastes by diffusion dialysis for systems containing $\mathrm{HCl}$ in the presence of sodium, copper, zinc, iron, nickel and

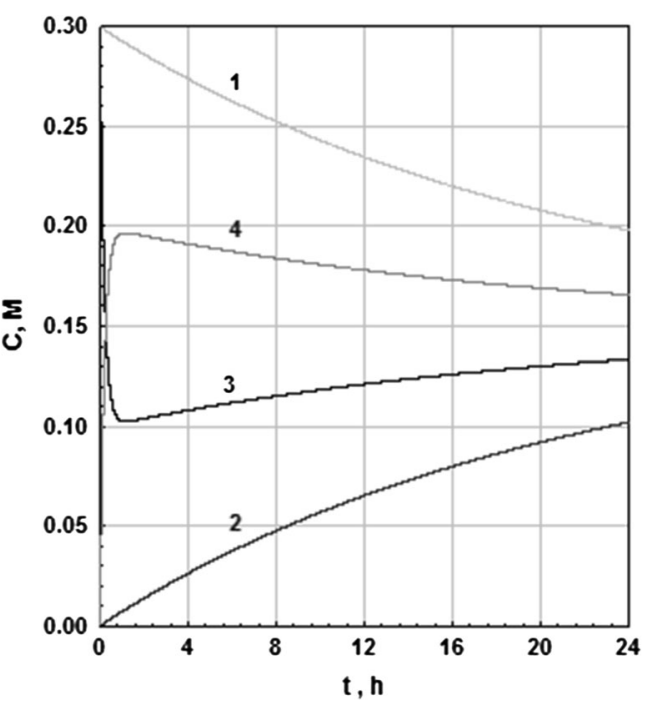

Fig. 1 Concentration dependencies $C$ of $\mathrm{Na}^{+}$cations (curves 1 and 2) and $\mathrm{K}^{+}$cations (curves 3 and 4 ) on time $t$ in the solutions in the $e$-cell (curves 1 and 3 ) and $i$-cell (curves 2 and 4). For both substances (KR and NaR), the initial total concentrations in the solution in the $e$-cell are $0.3 \mathrm{M}$ and the permeability coefficients are $600 \mathrm{dm} / \mathrm{h}$. The dissociation constants of $\mathrm{KR}$ and $\mathrm{NaR}$ are $10^{2} \mathrm{M}\left(k_{1}=10^{5} \mathrm{~h}^{-1}, k_{-1}=10^{3} \mathrm{M}^{-1} \mathrm{~h}^{-1}\right.$ and $10^{4} \mathrm{M}$ $\left(k_{2}=10^{5} \mathrm{~h}^{-1}, k_{-2}=10 \mathrm{M}^{-1} \mathrm{~h}^{-1}\right)$, respectively. The solution volumes in the $e$ - and $i$-cells are equal $\left(V_{i}=V_{e}\right) . S=1 \mathrm{dm}^{2}$

aluminium chlorides. For this purpose, only the temporal dependences of the concentration of ions in solutions separated by a membrane were used. Our model should be especially effective for studies where the initial part of the process is important and where it is necessary to take into account the dissociation of salts and the change in the volume of solutions during the transfer of substances through the membrane.

Let us demonstrate one more effect that can manifest itself at the membrane separation of substances differing in the degree of dissociation. Let us put a solution with the same concentration $(0.3 \mathrm{M})$ of substances KR and $\mathrm{NaR}$ into the $e$-cell, while distilled water is placed in the $i$-cell. The permeability coefficients of these substances are the same $(600 \mathrm{dm} / \mathrm{h})$; however, the degree of $\mathrm{NaR}$ dissociation is $10^{4} \mathrm{M}$, while that of $\mathrm{KR}$ is $10^{2} \mathrm{M}$. It should be stressed that there are no nonpenetrating substances in the given system, and both substances penetrate through the membrane with the same rate. All the previously developed theories of membrane transport (in particular, the Donnan theory) cannot be used for the description and investigation of such systems.

The temporal variation of concentrations of $\mathrm{Na}^{+}$and $\mathrm{K}^{+}$ cations in solutions separated by a membrane is shown in Fig. 1. One can see that a difference only in the degree of dissociation of the given substances enables their effective separation. However, the optimum separation conditions should be determined for this, since the separation of the given substrates does not occur under equilibrium: they are distributed in solutions on each side of the membranes with the same concentrations $(0.15 \mathrm{M})$. 
However, one can see from Fig. 1 that $1.16 \mathrm{~h}$ after starting the separation process the maximum $\mathrm{K}^{+}$amount -22.7 times bigger than $\mathrm{Na}^{+}$(i.e., 95.6\%) - appears in the solution in the $i$ cell. In this case, the $\mathrm{K}^{+}$concentration exceeds its equilibrium concentration and is $196.7 \mathrm{mM}$ (it should be noted that for $0.9 \mathrm{~h}-$ from $0.26 \mathrm{~h}$ to $1.16 \mathrm{~h}-\mathrm{K}^{+}$cations are transported against their concentration gradient). This effect does not appear if the dissociation constants of both substances are equal. In this case, the ratio between the concentrations of cations remains equal to one during the whole process.

The smaller the elapsed time from the start of the separation process (relative to $1.16 \mathrm{~h}$ ), the higher the content of $\mathrm{K}^{+}$cations (relative to the total amount of ions) in solution in the $i$-cell and the lower its concentration. For example, after $0.5 \mathrm{~h}$ the $\mathrm{K}^{+}$concentration is $184.6 \mathrm{mM}-44.8$ times higher than the $\mathrm{Na}^{+}$concentration (i.e., there is about $2 \% \mathrm{Na}^{+}$cations in the solution).

The greater the elapsed time from the start of the separation process (relative to $1.16 \mathrm{~h}$ ), the lower the content of $\mathrm{K}^{+}$cations in solution in the $i$-cell and the lower its concentration. For example, after $5 \mathrm{~h}$ the $\mathrm{K}^{+}$concentration is $189.2 \mathrm{mM}$ - only 5.9 times higher than the $\mathrm{Na}^{+}$concentration (i.e., there is about $17 \% \mathrm{Na}^{+}$cations in the solution).

When analysing the data presented in Fig. 1, one may conclude that the relative penetrating speed of $\mathrm{K}^{+}$cations through the membrane is much higher than that of $\mathrm{Na}^{+}$cations. However, this is due to the difference in the concentrations of undissociated molecules in solution in the $e$-cell rather than the permeability coefficients. So, at the moment $t=0$, the solution in the $e$-cell contains $1.78 \mathrm{mM}$ of undissociated $[K R]$ molecules and $0.0179 \mathrm{mM}$ of undissociated $[\mathrm{NaR}]$ molecules. Due to the hundred-fold difference in concentrations, even at the same penetrating speed through the membrane, a hundred times more $\mathrm{K}^{+}$ ions (as compared to $\mathrm{Na}^{+}$ions) pass through at the starting moment. This creates the illusion that $\mathrm{K}^{+}$ions penetrate through the membrane more quickly than $\mathrm{Na}^{+}$ions.

The above example shows that even a difference in the dissociation degree of two substances is sufficient for their membrane separation. This phenomenon may possibly be physiologically important because the relative penetrating speed of ions through a membrane can be regulated by a protein that can bind ions with different dissociation constants.

In addition to the specific examples of the application of the proposed kinetic model presented above, it can be also used for other purposes. It can be used to simulate processes whose driving force is a concentration gradient, for example, for dialysis. It should be noted again that the developed model, presented as a system of differential Eqs. (11-20), is only a particular example showing how to build a kinetic model in the general case based on a new principle and show its capabilities. Of course, to simulate a specific process, it is necessary to create a system of equations that considers all the components involved in the studied process.
The advantage of the model, in our opinion, is that most of the constants are rate constants of the dissociation of molecules into ions and ion associations into molecules. The values of many of these constants are known or can be determined in independent experiments. The nature of membranes can affect only the permeability coefficients of substances, which can be determined by comparing experimental results with model calculations (Luo et al. 2013). It is also possible to make the model more complex in order to associate the value of permeability coefficients to membrane properties.

The importance of our approach for the simulation of membrane transport is that it enhances our understanding of the physical processes of the transport of various substances through a semipermeable membrane. It provides opportunities for improving existing models of membrane processes, developing new models based on different principles, and allows looking from a different perspective at the processes occurring in living organisms with the participation of biological membranes.

\section{Conclusions}

We have developed a model to calculate the kinetics of the redistribution of ions and molecules through a semipermeable membrane in complex mixtures of substances penetrating and nonpenetrating through a membrane. It takes into account the degree of dissociation of these substances, their initial (total) concentrations in solutions separated by a membrane, and the volumes of these solutions.

Contrary to the Donnan model based on the assumption that only ions can penetrate through a membrane, our model is based on the assumption that uncharged particles - molecules or ion pairs - diffuse through a membrane.

Using our model, it is possible to calculate the temporal variation of the concentration of each reaction component in the system from the initial state to equilibrium. The state of equilibrium comes when the concentrations of penetrating undissociated molecules on each side of the membrane become equal. The Donnan distribution results directly from the kinetic calculation of the process of reaching equilibrium.

The Donnan effect results from the dissociation and equality of concentrations of molecules or ion pairs on each side of the membrane at equilibrium as well as from Le Chatelier's principle related to the presence of common ions of the substances penetrating and nonpenetrating through a membrane.

It is shown that only a slight difference between the degrees of dissociation of two substances can be used for their membrane separation.

It is also shown that the Donnan distribution (irregularity of ion distribution) and accordingly absolute value of the Donnan membrane potential increases if: (i) the nonpenetrating salt concentration (in one of the solutions) and its dissociation constant increases, (ii) the total penetrating 
salt concentration and its dissociation constant decreases, and (iii) the volumes ratio increases (between solutions with and without a nonpenetrating substance).

Name of conference at which the work was originally presented XIX International Conference "Dynamical system modelling and stability investigation" (DSMSI-2019), May 22-24, 2019, Kyiv, Ukraine.

Funding This research did not receive any specific grant from funding agencies in the public, commercial, or not-for-profit sectors.

\section{Compliance with ethical standards}

Conflict of interest The authors declare that they have no conflict of interest.

Ethical approval This article does not contain any studies with human participants or animals performed by any of the authors.

\section{Appendix}

We will show that our kinetic model based on the assumption that only molecules (uncharged particles) penetrate through a membrane, and the Donnan model, based on the assumption that only ions can penetrate through a membrane, are equivalent. For this purpose, we use the "classical" thermodynamic approach and consider two states of the membrane system: the initial and equilibrium states.

In the initial state, the substance $\mathrm{KB}$ at concentration $C_{1}$ and infinitely large dissociation constant $K_{1}$, is located in the $i$ cell (i.e., only $\mathrm{K}^{+}$and $\mathrm{B}^{-}$ions are present) while salt $\mathrm{KA}$ with concentration $C_{2}$ and relatively small dissociation constant $K_{2}$ is located in the $e$-cell. It should be taken into account that $\mathrm{K}^{+}$ and $\mathrm{A}^{-}$ions are in the $i$-cell simultaneously with the undissociated KA molecules. The cell volumes are equal to $V_{i}=V_{e}$.

To calculate the concentrations of ions and molecules in the $e$-cell, we designate $z=\left[K^{+}\right]=\left[A^{-}\right]$and write down an equation for the dissociation constant $K_{2}$ (taking into account Eq. $\stackrel{(8)):}{K_{2}}=\frac{z^{2}}{C_{2}-z}$
Solving the quadratic equation in $z$, we get:

$z=\frac{-K_{2}+\sqrt{K_{2}^{2}+4 K_{2} C_{2}}}{2}$

Thereby the initial state of the system under investigation may be presented as:

$$
\begin{aligned}
& {\left[K^{+}\right]_{i}=} C_{1} \mid\left[K^{+}\right]_{e}=\frac{-K_{2}+\sqrt{K_{2}^{2}+4 K_{2} C_{2}}}{2} . \\
& {\left[B^{-}\right]_{i}=} C_{1} \mid\left[A^{-}\right]_{e}=\frac{-K_{2}+\sqrt{K_{2}^{2}+4 K_{2} C_{2}}}{2} \\
& \mid[K A]_{e}=\frac{2 C_{2}+K_{2}-\sqrt{K_{2}^{2}+4 K_{2} C_{2}}}{2}
\end{aligned}
$$

Firstly, we will calculate the equilibrium state using the Donnan approach, i.e. we assume that only $\mathrm{K}^{+}$and $\mathrm{A}^{-}$ions penetrate through the membrane.

As a result of permeable $\mathrm{K}^{+}$and $\mathrm{A}^{-}$ions passing through the membrane, some amount of salt moves from the $e$-cell into the $i$-cell, taking into account that the electroneutrality condition requires transferring equal amounts of $\mathrm{K}^{+}$and $\mathrm{A}^{-}$ions. As a result, the total salt concentration in the $e$-cell decreases by $x$ and is then $C_{2}-x$. In accordance with Eq. (A1) in which $C_{2}-x$ has to be written in the denominator instead of $C_{2}$, it is possible to calculate the concentrations of ions and molecules in the $e$-cell at equilibrium.

Some of the ions that penetrate into the $i$-cell form molecules whose concentration is denoted by $y$. Then the concentrations of $\mathrm{K}^{+}$cations and $\mathrm{A}^{-}$anions may be written as $C_{1}+x-y$ and $x-y$, respectively. The concentrations of ions and undissociated molecules are related by the following equation:

$K_{2}=\frac{\left(C_{1}+x-y\right)(x-y)}{y}$

with which $y$ can be calculated:

$y=\frac{\left(C_{1}+K_{2}\right)+2 x-\sqrt{\left(C_{1}+K_{2}\right)^{2}+4 x K_{2}}}{2}$

Thereby the equilibrium state of the system under investigation may be presented as:

$$
\begin{array}{ll}
{\left[K^{+}\right]_{i}=\frac{C_{1}-K_{2}+\sqrt{\left(C_{1}+K_{2}\right)^{2}+4 x K_{2}}}{2}} & \mid\left[K^{+}\right]_{e}=\frac{-K_{2}+\sqrt{K_{2}^{2}+4 K_{2}\left(C_{2}-x\right)}}{2} \\
{\left[A^{-}\right]_{i}=\frac{-C_{1}-K_{2}+\sqrt{\left(C_{1}+K_{2}\right)^{2}+4 x K_{2}}}{2}} & \mid\left[A^{-}\right]_{e}=\frac{-K_{2}+\sqrt{K_{2}^{2}+4 K_{2}\left(C_{2}-x\right)}}{2} \\
{[K A]_{i}=\frac{\left(C_{1}+K_{2}\right)+2 x-\sqrt{\left(C_{1}+K_{2}\right)^{2}+4 x K_{2}}}{2}} & \mid[K A]_{e}=\frac{2\left(C_{2}-x\right)+K_{2}-\sqrt{K_{2}^{2}+4 K_{2}\left(C_{2}-x\right)}}{2} \\
{\left[B^{-}\right]_{i}=C_{1}} & \mid
\end{array}
$$


Now, let us assume that only undissociated KA molecules penetrate through the membrane like in our kinetic approach. In the process of transition of the system from the initial state to the equilibrium state, the total salt concentration in the $e$-cell will decrease by $x$, and these $x$ moles of salt KA will move to the solution located in the $i$-cell. In this case, the concentrations of ions and molecules in the $e$-cell at equilibrium are as follows:

$\begin{aligned} {\left[K^{+}\right]_{e} } & =\left[A^{-}\right]_{e}=\frac{-K_{2}+\sqrt{K_{2}^{2}+4 K_{2}\left(C_{2}-x\right)}}{2} \\ {[\mathrm{KA}]_{e} } & =\frac{2\left(C_{2}-x\right)+K_{2}-\sqrt{K_{2}^{2}+4 K_{2}\left(C_{2}-x\right)}}{2}\end{aligned}$

Some KA molecules that have moved to the $i$-cell dissociate into ions. Let us denote the concentration of resulting ions as $y$. Then the concentrations of $\mathrm{K}^{+}$cations and $\mathrm{A}^{-}$anions may be written as $C_{1}+y$ and $y$, respectively The concentrations of ions and undissociated molecules are related by the following equation:

$K_{2}=\frac{y\left(C_{1}+y\right)}{x-y}$

with which $y$ can be calculated:

$y=\frac{-\left(C_{1}+K_{2}\right)+\sqrt{\left(C_{1}+K_{2}\right)^{2}+4 x K_{2}}}{2}$

The resulting expression is the concentration of the $\mathrm{A}^{-}$ anion in $i$-cell $\left(\left[A^{-}\right]_{i}\right)$. Now, it is possible to calculate $\left[K^{+}\right]_{i}$ and $[K A]_{i}$ :

$\left[K^{+}\right]_{i}=C_{1}+y=\frac{C_{1}-K_{2}+\sqrt{\left(C_{1}+K_{2}\right)^{2}+4 x K_{2}}}{2}$

$[\mathrm{KA}]_{i}=x-y=\frac{\left(C_{1}+K_{2}\right)+2 x-\sqrt{\left(C_{1}+K_{2}\right)^{2}+4 x K_{2}}}{2}$

As you can see, both approaches lead to identical results, suggesting their equivalence. Thus, regardless of whether undissociated molecules or individual ions are transported through the membrane (in quantities guaranteeing the maintenance of electrical neutrality), the system reaches the same equilibrium state (from an identical initial state).

We also show that both approaches lead to the equality of equilibrium concentrations of undissociated salt molecules in solutions separated by a semipermeable membrane.

At equilibrium, the penetrating ions obey the Donnan distribution, so the following equations may be written:

$\lambda=\frac{\left[K^{+}\right]_{i}}{\left[K^{+}\right]_{e}}=\frac{C_{1}-K_{2}+\sqrt{\left(C_{1}+K_{2}\right)^{2}+4 x K_{2}}}{-K_{2}+\sqrt{K_{2}^{2}+4 K_{2}\left(C_{2}-x\right)}}$

$$
\lambda=\frac{\left[A^{-}\right]_{e}}{\left[A^{-}\right]_{i}}=\frac{-K_{2}+\sqrt{K_{2}^{2}+4 K_{2}\left(C_{2}-x\right)}}{-C_{1}-K_{2}+\sqrt{\left(C_{1}+K_{2}\right)^{2}+4 x K_{2}}}
$$

Let us compare the concentrations of undissociated KA molecules in the $i$ - and $e$-cells:

$$
\frac{[K A]_{i}}{[K A]_{e}}=\frac{\left(C_{1}+K_{2}\right)+2 x-\sqrt{\left(C_{1}+K_{2}\right)^{2}+4 x K_{2}}}{2\left(C_{2}-x\right)+K_{2}-\sqrt{K_{2}^{2}+4 K_{2}\left(C_{2}-x\right)}}
$$

It is difficult to determine from Eq. (A13) if the above ratio equals one. So let us focus on Eqs. (A11) and (A12): their ratio equals one.

$$
\frac{\left[K^{+}\right]_{i}\left[A^{-}\right]_{i}}{\left[K^{+}\right]_{e}\left[A^{-}\right]_{e}}=\frac{\left(C_{1}-K_{2}+\sqrt{\left(C_{1}+K_{2}\right)^{2}+4 x K_{2}}\right)\left(-C_{1}-K_{2}+\sqrt{\left(C_{1}+K_{2}\right)^{2}+4 x K_{2}}\right)}{\left(-K_{2}+\sqrt{K_{2}^{2}+4 K_{2}\left(C_{2}-x\right)}\right)\left(-K_{2}+\sqrt{K_{2}^{2}+4 K_{2}\left(C_{2}-x\right)}\right)}=1
$$

After transformations we get:

$\frac{\left[K^{+}\right]_{i}\left[A^{-}\right]_{i}}{\left[K^{+}\right]_{e}\left[A^{-}\right]_{e}}=\frac{2 K_{2}\left[\left(C_{1}+K_{2}\right)+2 x-\sqrt{\left(C_{1}+K_{2}\right)^{2}+4 x K_{2}}\right]}{2 K_{2}\left[2\left(C_{2}-x\right)+K_{2}-\sqrt{K_{2}^{2}+4 K_{2}\left(C_{2}-x\right)}\right]}=1$

The comparison between Eqs. (A13) and (A15) clearly shows that at equilibrium the concentrations of undissociated molecules in the cells separated by a membrane are equal:

$\frac{[K A]_{i}}{[K A]_{e}}=\frac{\left[K^{+}\right]_{i}\left[A^{-}\right]_{i}}{\left[K^{+}\right]_{e}\left[A^{-}\right]_{e}}=1$

\section{References}

Al-Obaidi MA, Kara-Zaitri C, Mujtaba IM (2017) Scope and limitations of the irreversible thermodynamics and the solution diffusion models for the separation of binary and multi-component systems in reverse osmosis process. Comput Chem Eng 100:48-79. https:// doi.org/10.1016/j.compchemeng.2017.02.001

Cohen H, Cooley JW (1965) The numerical solution of the timedependent Nernst-Planck equations. Biophys J 5:145-162. https:// doi.org/10.1016/S0006-3495(65)86707-8

Davis TA (2000) Donnan dialysis. In: Wilson ID, Adlard ER, Cooke M, Poole CF (eds) Encyclopedia of separation science. Academic Press, London, pp 1701-1707

Déon S, Escoda A, Fievet P, Salut R (2013) Prediction of single salt rejection by NF membranes: an experimental methodology to assess physical parameters from membrane and streaming potentials. Desalination 315:37-45. https://doi.org/10.1016/j.desal.2012.09. 005

Donnan FG (1924) The theory of membrane equilibria. Chem Rev 1:7390. https://doi.org/10.1021/cr60001a003

Donnan FG (1995) Theory of membrane equilibria and membrane potentials in the presence of non-dialysing electrolytes. A contribution to physical-chemical physiology. J Membr Sci 100:45-55. https:// doi.org/10.1016/0376-7388(94)00297-C 
Duffey ME, Fennell Evans D, Cussler EL (1978) Simultaneous diffusion of ions and ion pairs across liquid membranes. J Membr Sci 3:1-14. https://doi.org/10.1016/S0376-7388(00)80407-X

Fridman-Bishop N, Tankus KA, Freger V (2018) Permeation mechanism and interplay between ions in nanofiltration. J Membr Sci 548:449 458. https://doi.org/10.1016/j.memsci.2017.11.050

Galach M, Waniewski J (2012) Membrane transport of several ions during peritoneal dialysis: mathematical modeling. Artif Organs 36: E163-E178. https://doi.org/10.1111/j.1525-1594.2012.01484.x

Galama AH, Post JW, Hamelers HVM, Nikonenko VV, Biesheuvel PM (2016) On the origin of the membrane potential arising across densely charged ion exchange membranes: how well does the TeorellMeyer-Sievers theory work? J Membr Sci Res 2:128-140. https:// doi.org/10.22079/jmsr.2016.20311

Gimmi T, Alt-Epping P (2018) Simulating Donnan equilibria based on the Nernst-Planck equation. Geochim Cosmochim Acta 232:1-13. https://doi.org/10.1016/j.gca.2018.04.003

Grzegorczyn S, Ślęzak A (2006) Time characteristics of electromotive force in single-membrane cell for stable and unstable conditions of reconstructing of concentration boundary layers. J Membr Sci 280: 485-493. https://doi.org/10.1016/j.memsci.2006.02.004

Higa M, Kira A (1992) Theory and simulation of ion transport in nonstationary states against concentration gradients across ion-exchange membranes. J Phys Chem 96:9518-9523. https://doi.org/10.1021/ j100202a081

Johnson KS, Pytkowicz RM (1978) Ion association of $\mathrm{Cl}^{-}$with $\mathrm{H}^{+}, \mathrm{Na}^{+}$, $\mathrm{K}^{+}, \mathrm{Ca}^{2+}$, and $\mathrm{Mg}^{2+}$ in aqueous solutions at $25^{\circ} \mathrm{C}$. Am J Sci 278 : 1428-1447. https://doi.org/10.2475/ajs.278.10.1428

Kim DY, Lee MH, Boram G, Kim JH, Lee S, Yang DR (2010) Modeling of solute transport in multi-component solution for reverse osmosis membranes. Desalination Water Treat 15:20-28. https://doi.org/10. 5004/dwt.2010.1662

Kondepudi D, Prigogine I (1998) Modern thermodynamics. From heat engines to dissipative structures. John Wiley \& Sons, New York

Kosterin SA, Cherny AP (1991) Gibbs-Donnan equilibrium in the system membrane vesicules - incubation medium. Biofizika 36:826-829. (In Russian)

Kozmai A, Chérif M, Dammak L, Bdiri M, Larchet C, Nikonenko V (2017) Modelling non-stationary ion transfer in neutralization dialysis. J Membr Sci 540:60-70. https://doi.org/10.1016/j.memsci. 2017.06.039

Kumaran M, Bajpai S (2015) Application of extended Nernst Planck model in nano filtration process -a critical review. Int J Eng Res Rev 3:40-49

Kurbel S (2011) Donnan effect on chloride ion distribution as a determinant of body fluid composition that allows action potentials to spread via fast sodium channels. Theor Biol Med Model 8:16. https://doi.org/10.1186/1742-4682-8-16

Lang GE, Stewart PS, Vella D, Waters SL, Goriely A (2014) Is the Donnan effect sufficient to explain swelling in brain tissue slices? J Roy Soc Interface 11:20140123. https://doi.org/10.1098/rsif.2014. 0123

Luo J, Wu C, Wu Y, Xu T (2013) Diffusion dialysis of hydrochloric acid with their salts: effect of co-existence metal ions. Sep Purif Technol 118:716-722. https://doi.org/10.1016/j.seppur.2013.08.014

Marcus Y, Hefter G (2006) Ion pairing. Chem Rev 106:4585-4621. https://doi.org/10.1021/cr040087x

Mazur I, Kosterin S, Veklich T, Shkrabak O (2014) Gibbs-Donnan potential as a tool for membrane vesicles polarization. J Biophys Chem 5:78-89. https://doi.org/10.4236/jbpc.2014.52009

Moshtarikhah S, Oppers NAW, de Groot MT, Keurentjes JTF, Schouten JC, van der Schaaf J (2017) Nernst-Planck modeling of multicomponent ion transport in a Naflon membrane at high current density. $\mathrm{J}$ Appl Electrochem 47:51-62. https://doi.org/10.1007/s10800-016$1017-2$
Neihof R, Sollner K (1957) The transitory overshooting of final equilibrium concentrations in membrane systems which drift toward the Gibbs-Donnan membrane equilibrium. J Phys Chem 61:159-163. https://doi.org/10.1021/j150548a008

Nguyen MK, Kurtz I (2006) Quantitative interrelationship between Gibbs-Donnan equilibrium, osmolality of body fluid compartments, and plasma water sodium concentration. J Appl Physiol 100:12931300. https://doi.org/10.1152/japplphysiol.01274.2005

Nouri S, Dammak L, Bulvestre G, Auclair B (2002) Studies of the crossed ionic fluxes through a cation-exchange membrane in the case of Donnan dialysis. Desalination 148:383-388. https://doi. org/10.1016/S0011-9164(02)00734-8

Osterhout WJV (1925) Is living protoplasm permeable to ions? J Gen Physiol 8:131-146. https://doi.org/10.1085/jgp.8.2.131

Osterhout WJV (1929) The kinetics of penetration. J Gen Physiol 13: 261-294. https://doi.org/10.1085/jgp.13.2.261

Palmeri J, Lefebvre X (2006) Computer simulation of Nanofiltration, membranes and processes. In: Rieth M, Schommers W (eds) Handbook of theoretical and computational nanotechnology, Transport Phenomena and Nanoscale Processes, vol 5, 1st edn. American Scientific Publishers, Stevenson Ranch, pp 93-214

Philipse A, Vrij A (2011) The Donnan equilibrium: I. on the thermodynamic foundation of the Donnan equation of state. J Phys Condens Matter 23:194106. https://doi.org/10.1088/0953-8984/23/19/ 194106

Prado-Rubio OA, Møllerhøj M, Jørgensen SB, Jonsson G (2010) Modeling Donnan dialysis separation for carboxylic anion recovery. Comput Chem Eng 34:1567-1579. https://doi.org/10.1016/j. compchemeng.2010.03.003

Pyrzynska K (2006) Preconcentration and recovery of metal ions by Donnan dialysis. Microchim Acta 153:117-126. https://doi.org/10. 1007/s00604-005-0434-4

Ramirez P, Alcaraz A, Mafe S, Pellicer J (2002) Donnan equilibrium of ionic drugs in $\mathrm{pH}$-dependent fixed charge membranes: theoretical modelling. J Colloid Interface Sci 253:171-179. https://doi.org/10. $1006 /$ jcis. 2002.8508

Rohman FS, Aziz N (2008) Mathematical model of ion transport in electrodialysis process. Bull Chem React Eng Catal 3:3-8. https://doi. org $/ 10.9767 /$ bcrec.3.1-3.7122.3-8

Sarkar S, Sengupta A, Prakash P (2010) The Donnan membrane principle: opportunities for sustainable engineered processes and materials. Environ Sci Technol 44:1161-1166. https://doi.org/10.1021/ es 9024029

Shu L, Liu X, Li Y, Yang B, Huang S, Lin Y, Jin S (2016) Modified Kedem-Katchalsky equations for osmosis through nano-pore. Desalination 399:47-52. https://doi.org/10.1016/j.desal.2016.08. 011

Sobana S, Panda RC (2011) Review on modelling and control of desalination system using reverse osmosis. Rev Environ Sci Biotechnol 10:139-150. https://doi.org/10.1007/s11157-011-9233-z

Steele A, Arias J (2014) Accounting for the Donnan effect in diafiltration optimization for high concentration UFDF applications. BioProcess Int 12:50-54

Szczepański P, Szczepańska G (2017) Donnan dialysis - a new predictive model for non-steady state transport. J Membr Sci 525:277-289. https://doi.org/10.1016/j.memsci.2016.11.017

Tanaka Y (2012) Measurement of membrane characteristics using the phenomenological equation and the overall mass transport equation in ion-exchange membrane electrodialysis of saline water. Int $\mathrm{J}$ Chem Eng 2012:Article ID 148147, 12. https://doi.org/10.1155/ 2012/148147

Tian H, Zhang L, Wang M (2015) Applicability of Donnan equilibrium theory at nanochannel-reservoir interfaces. J Colloid Interface Sci 452:78-88. https://doi.org/10.1016/j.jcis.2015.03.064 
Vega FA, Weng L, Temminghoff EJM, Van Riemsdijk WH (2010) Donnan membrane technique (DMT) for anion measurement. Anal Chem 82:2932-2939. https://doi.org/10.1021/ac9029339

Volpert AI, Hudyaev SI (1975) Analyses in classes of discontinuous functions and equations of mathematical physics. Nauka, Moscow. (In Russian)

Wang J, Dlamini DS, Mishra AK, Pendergast MTM, Wong MCY, Mamba BB, Freger V, Verliefde ARD, Hoek EMV (2014) A critical review of transport through osmotic membranes. J Membr Sci 454: 516-537. https://doi.org/10.1016/j.memsci.2013.12.034

Yaroshchuk A, Martínez-Lladó X, Llenas L, Rovira M, de Pablo J (2011) Solution-diffusion-film model for the description of pressure-driven trans-membrane transfer of electrolyte mixtures: one dominant salt and trace ions. J Membr Sci 368:192-201. https://doi.org/10.1016/j. memsci.2010.11.037

Zhao R, Van Soestbergen M, Rijnaarts HHM, Van der Wal A, Bazant MZ, Biesheuvel PM (2012) Time-dependent ion selectivity in capacitive charging of porous electrodes. J Colloid Interface Sci 384:38-44. https://doi.org/10.1016/j.jcis.2012.06.022

Publisher's Note Springer Nature remains neutral with regard to jurisdictional claims in published maps and institutional affiliations. 